\title{
LXI. Elementary notes on thermodynamics: the plug experiment
}

\section{Edgar Buckingham}

To cite this article: Edgar Buckingham (1906) LXI. Elementary notes on thermodynamics: the plug experiment, Philosophical Magazine Series 6, 11:65, 678-685, DOI: $10.1080 / 14786440609463486$

To link to this article: http://dx.doi.org/10.1080/14786440609463486

曲 Published online: 16 Apr 2009.

Submit your article to this journal $₫$

Џ Article views: 2

Q View related articles $₫$ 
over the one first described and has several inberent disadvantages, it was discarded in favour of what might be called the series arrangement.

In conclusion, the author wishes to thank Dr. J. A. Fleming, F.R.S, for his kindness in permiting him to publish the results of these experiments, which were carried out under his direction at the Pender Laboratory, University College, London.

\section{Elementary Notes on Thermodynamics: the Plug} Experiment. By EDgar BUCkingham*.

$\S 1$. THEORETICALLY there are many ways of establishing the thermodynamic scale of temperature, but practically, experiments on the properties of gases offer the only mode of attacking the problem that has been followed with any success. For this purpose, what we need to know is the manner in which the internal energy of the gas used in our standard gas-thermometer varies with the volume, when the temperature is constant; and for the most precise information on this point, we have, at present, to refer to the porous-plug experiment or something equivalent to it.

The use of reasoning based on the second law of thermodynamics is to be met with very frequently; and we find, in almost every case, that it involves the assumption that the international hydrogen scale, to which the observations are referred, differs only by an additive constant from Lord Kelvin's thermodynamic scale which appears in the thermodynamic formulæ. It might be expected, therefore, that a clear treatment of the plug experiment would be found in every text-book of thermodynamics, but that expectation is not justified. Some of our best standard works hardly mention the plug experiment, and the general impression one gets from most of the books I have examined, is that the authors consider the subject either too difficult to be discussed or so simple as to deserve only casual notice. I think, however, that a clear and simple treatment of the theory of some method or methods by which the relation of the gas scale to the thermodynamic seale might be found, is distinctly worth while, even if there is nothing new about the discussion except, possibly, the form in which it is put.

$\$ 2$. Beside the original Joule-Thomson form of the plug experiment, in which the flow of gas through the porous

* Comnunicated by the Author. 
plug is adiabatic*, I shall consider a modified scheme, in which energy is supplied to the gas, during its passage through the plug, at such a rate as to make the flow isothermal + ; and, furthermore, I shall consider a simplified form of Gay-Lussac's and Joule's free expansion experiments, in which heat is supposed to be supplied or withdrawn during the free expansion so as to to make it isothermal $\neq$.

The fundamental equations for these three experiments are very easy to deduce, if one starts by having a clear diagram.

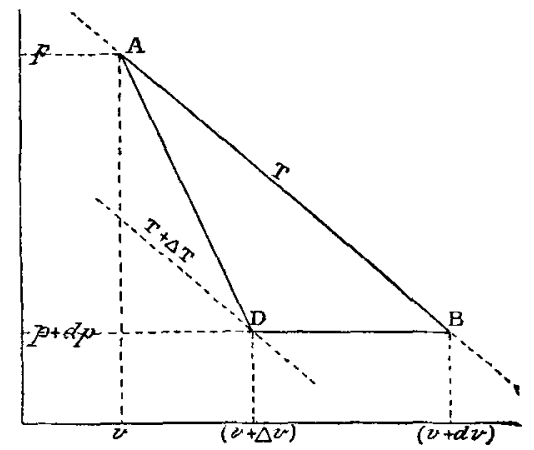

Let A (coordinates : $p, v, \mathrm{~T}$ ) represent the initial state of

* Objection has been made to calling the flow adiabatic, because heat is generated mechanically in the plug. When we consider that this heat is probably mainly, if not entirely, due to viscosity, and if so, is developed in the gas itself, and not generated in the solid material of the plug by true friction and then communicated to the gas, the objection does not seem well founded. The term "adiabatic plug experiment" is, at all events, a convenient designation for the Joule-Thomson experiment as distinguished from the proposed isothermal form.

+ Phil. Mag. October 1903.

I At first sight, it seems doubtful whether we are justified in applying thermodynamic reasoning to an essentially tumultuous process such as the free expansion of a gas; for the use of the two laws in their usual elementary form implies that the system under consideration has a definite temperature, whereas during a tumultuous process, the gas cannot be said to have any definite temperature at all. After the tumultuous motions have subsided and the gas has come to a state of internal equilibrium, both mechanical and thermal, it has again a definite temperature. The reasoning by which, from the assumption of the nonexistence of infinite sources and sinks of energy, we establish the existence of the internal energy, $\epsilon$, as a function of the instantaneous coordinates of the system, makes no assumption regarding the nature of the path by which any state of the system may be reached from any other, but assumes only that we may always, by some means or other, make the system pass from any possible state to any other. Hence it is entirely legitimate to use the first law-the energy law-in comparing any two states, regardless of the nature of the process by which one has actually been reached from the other, provided it be not in the nature of things impossible to make the system pass again by sowe path or other from the final to the initial state. 
unit mass of the gas. Let $\mathrm{D}$ (coordinates : $p+d p, v+\Delta v$, $\mathrm{T}+\Delta \mathrm{T}$ ) represent the state after the irreversible adiabatic flow through the porous plug. Let $\mathrm{B}$ (coordinates : $p+d p$, $v+d v, \mathrm{~T}$ ) represent the state the gas would have been in, if the same fall of pressure had occurred without change of temperature. The temperature $\mathrm{T}$ is to be understood as measured on the scale of a constant-pressure thermometer filled with the gas under investigation.

$\S 3$. Consider first the Joule-Thomson, or adiabatic, plug experiment. If the state of flow is steady and no exchange of heat with other bodies than the plug is taking place, the change of internal energy of unit mass of gas in passing through the plug is

$$
\epsilon_{2}-\epsilon_{1}=p_{1} v_{1}-p_{2} v_{2}:
$$

or, if we let the fall of pressure be infinitesimal,

$$
\Delta \epsilon=-\Delta(p v)=-p \Delta v-v d p, . . .
$$

$\Delta p$ and $d p$ being identical, as is seen from the figure. Let the gas which has thus passed from the state $A$ to the state D be now brought, at constant pressure, to the state $\mathrm{B}$. If $d \varepsilon$ be the excess of the specific internal energy at $B$ over that at $A$, we now have

$$
d \epsilon=\Delta \epsilon+Q+W,
$$

in which $\mathrm{Q}$ and $\mathrm{W}$ are, respectively, the heat added to and the work done on the unit mass of gas during the isopiestic change DB, which brings it back from $(\mathrm{T}+\Delta \mathrm{T})$ to its original temperature T. We suppose, for simplicity, that all quantities of heat are expressed in ergs.

If we let $\mu$ be the ratio of the fall of temperature to the fall of pressure, i.e. $\Delta \mathrm{T} / d p$, we have $\Delta \mathrm{T}=\mu d p$, and the value of $Q$ is evidently*

$$
\mathrm{Q}=-\mu \mathrm{C}_{p} d p, \quad \text {. . . . . . }
$$

$\mathrm{C}_{p}$ being the specific heat at constant pressure. The value of $W$ is given by the equation

We also have

$$
\mathrm{W}=-p(d v-\Delta v) . \quad . \quad . \quad . \quad .
$$

$$
d v-\Delta v=(-\Delta \mathrm{T})\left(\frac{\partial v}{\partial \mathrm{T}}\right)_{p}=-\mu d p\left(\frac{\partial v}{\partial \mathrm{T}}\right)_{p}, \ldots
$$

so that

$$
\Delta v=d v+\left(\frac{\partial v}{\partial \mathrm{T}}\right)_{p} \mu l p . \quad . \quad . \quad . \quad .
$$

* The figure as drawn corresponds to the ordinary case in which there is a fall of temperature during the flow through the plug. The sign of $\mu$, as here defined, is then positive, while $d p$ is negative, so that the value of $Q$ as given in (3) is in fact positive, as it is evident from the figure that it must be. 
By substituting in equation (2) the values given by equations (1), (3), (4), (5), and (6), we get

$$
d \epsilon=-p d v-v d p-\mu \mathrm{C}_{p} d p ;
$$

and since the differentials which appear in this equation all refer to the isothermal change $A B$, we may write it in the form *

$$
\left(\frac{\partial \epsilon}{\partial v}\right)_{\theta}=-p-\left(v+\mu \mathrm{C}_{p}\right)\left(\frac{\partial p}{\partial v}\right)_{\theta} \cdot \ldots .
$$

This equation is, of course, not new, but few writers of text-books on thermodynamics seem to think it worth deducing.

The equations for the other two experiments come out much more directly. In the case of the isothermal plug experiment, in which the gas passes at once from the state $A$ to the state $\mathrm{B}$, let $\rho$ be the ratio of the energy abstracted from the gas to the fall of pressure $d p$. Then we have, obviously,

$$
d \epsilon=-p d v-v d p+\rho d p, \text {. . . . . }
$$

or by the same considerations as before,

$$
\left(\frac{\partial \epsilon}{\partial v}\right)_{\theta}=-p-(v-\rho)\left(\frac{\partial p}{\partial v}\right)_{\theta} \cdot \ldots . .
$$

In the irreversible isothermal free expansion from $\mathrm{A}$ to $\mathrm{B}$, the external work is zero. If we let $\lambda$ be the ratio of the energy (heat) which must be added, per unit mass of gas, to the increase of specific volume $d v$, in order to make the final temperature of the gas, after its tumultuous motions have subsided, the same as its initial temperature, we have

$$
d \epsilon=\lambda d v, \quad \text {. . . . . . . }
$$

or

$$
\left(\frac{\partial \epsilon}{\partial v}\right)_{\theta}=\lambda . . \quad . \quad . \quad . \quad . \quad .
$$

$\S 4$. In deducing equations (A), (B), and (C), no use is made of the second law; hence they do not involve the thermodynamic temperature $\theta$ at all, and cannot, by themselves, tell us anything about it. But suppose, finally, that the gas passes from the state $A$ to the state $B$ by a reversible isothermal expansion. For any reversible change of state of a system having only two degrees of freedom and acted

* Constancy of temperature is indicated by the subseript $\theta:$ if $\theta$ is the thermodynamic temperature, $T$ is evidently constant whenever $\theta$ is constant.

Phil. Mag. S. 6. Vol. 11. No. 65. May 1906. 2 Y 
on by no external forces except a uniform normal pressure, we have

$$
d \epsilon=\theta d \eta-p d v, .
$$

which is merely a statement of the two laws of thermodynamies for such a system, $\eta$ representing the entropy. If the process is isothermal, we may write equation (10) in the form

$$
\left(\frac{\partial \epsilon}{\partial v}\right)_{\theta}=\theta\left(\frac{\partial \eta}{\partial v}\right)_{\theta}-p
$$

To give this a physical meaning we have to eliminate the entropy $\boldsymbol{\eta}$, by means of the familiar "thermodynamic relation "

$$
\left(\frac{\partial \eta}{\partial v}\right)_{\theta}=\left(\frac{\partial p}{\partial \theta}\right)_{v}
$$

thus obtaining the equation

$$
\left(\frac{\partial \epsilon}{\partial v}\right)_{\theta}=-p+\theta\left(\frac{\partial p}{\partial \theta}\right)_{v}
$$

$\S 5$. By comparing equation (D) with (A), (B), and (C) successively, we get three equations which, when slightly transformed by means of the relation

$$
\left(\frac{\partial p}{\partial v}\right)_{\theta}\left(\frac{\partial v}{\partial \theta}\right)_{p}\left(\frac{\partial \theta}{\partial p}\right)_{v}=-1
$$

may be written

$$
\begin{aligned}
& \theta\left(\frac{\partial v}{\partial \theta}\right)_{p}=v+\mu \mathrm{C}_{p}, \\
& \theta\left(\frac{\partial v}{\partial \theta}\right)_{p}=v-\rho, \ldots . \\
& \theta\left(\frac{\partial p}{\partial \theta}\right)_{v}=p+\lambda, \ldots . .
\end{aligned}
$$

and which give relations between $\theta$ and the data to be obtained from the three experiments. Expressing these in integral form we have

$$
\begin{aligned}
& \log \frac{\theta}{\theta_{0}}=\int_{v_{0}}^{v} \frac{d v}{v+\mu \mathrm{C}_{p}}(p=\pi=\text { constant }), . . \\
& \log \frac{\theta}{\theta_{0}}=\int_{v_{0}}^{v} \frac{d v}{v-\rho} \quad(p=\pi=\text { constant }), . . \\
& \log \frac{\theta}{\theta_{0}}=\int_{p_{0}}^{p} \frac{d p}{p+\lambda} \quad(v=\phi=\text { constant }), . .
\end{aligned}
$$


Equations (H) and (I) show how we may, if the experimental data are at hand, find the ratio of the numerical values of any two temperatures denoted by $\theta$ and $\theta_{0}$ on the thermodynamic scale, at which the specific volume of the gas has the values $v$ and $v_{0}$ at the constant pressure $\pi$. Equation $(J)$ gives us similar information in terms of pressure at the constant specific volume $\phi$. By using the equation $p v=\mathrm{RT}$ which defines the absolute temperature $\mathrm{T}$ of the gasthermometer, when either $p$ or $v$ is constant, it is, of course, easy to replace $v$ or $p$ by the absolute temperature measured by a constant-pressure or constant-volume thermometer filled with the gas in question.

These deductions, which make no pretence of novelty, are all quite simple enough to be put into the most elementary text-book of thermodynamics. In view of the difficulty students have in getting any clear ideas about the thermodynamic scale of temperature from Joule and Lord Kelvin's papers on the plug experiment, I think it would be well if some such simple discussion were more commonly given. For although this treatment is only formal, any one can see from the equations what experimental data are needed and how we must use them if we wish to go on to find actual numerical relations. The main thing is to have the conviction that there is a definite way in which the experimental data might be used if we had them, and anyone who has not attained that conviction by the "seeing-is-believing" process must sometimes feel rather at sea in using thermodynamics.

$\S 6$. In order actually to perform the integrations indicated in equations $(\mathrm{H}),(\mathrm{I})$, and $(\mathrm{J})$, we have, in the case of the adiabatic plug experiment, to know the values of $\mu$ and $\mathrm{C}_{p}$ as functions of $v$ for the given constant pressure used in our constant-pressure gas-thermometer ; and it may be noted that the value of Joule's equivalent is also involved, unless we have the values of $\mathrm{C}_{p}$ measured directly in ergs. For the isothermal plug experiment, we need merely the value of $\rho$ as a function of $v$ at the same constant pressure. By supplying this energy $\rho$ electrically, it may be measured in ergs as exactly as the absolnte accuracy of our electrical standards permits. This method would not serve us in the case of hydrogen above $-80^{\circ} \mathrm{C}$, nor for other gases above their, as yet unknown, inversion temperatures, though it is conceivable that in such cases the Peltier effect might be utilized for electric cooling. For the free expansion experiment the data needed are the values of $\lambda$ in terms of $p$ at the given specific volume used in the constant-volume gasthermometer. It may be remarked, in this connexion, that 
some degree of uncertainty must always attach to the substitution of experimental values of $\mu, \rho$, and $\lambda$ for differential coefficients : we can only hope that the extrapolation from finite to infinitesimal differences of pressure is allowable.

The quantity $\lambda$, or $(\partial \epsilon / \partial v)_{\theta}$, is useful for expressing the deviations of the behaviour of any real gas from the simple Boyle-GayLussac law, and it would be convenient to have values of $\lambda$ tabulated for the various gases. Such a tabulation has been given by Amagat* for carbonic acid and ethylene up to 1000 atmospheres, for several temperatures between $0^{\circ}$ and $200^{\circ} \mathrm{C}$.; also for oxygen, nitrogen, air, and hydrogen for the mean temperature of $50^{\circ} \mathrm{up}$ to 1000 atmospheres, and for the mean temperature of $25^{\circ}$ from 1000 to 4000 atmospheres. The computations were made by means of equation (G) from Amagat's measurements of $p$ and $(\partial p / \partial \theta)_{v}$, on the assumption that $\theta$ and $\mathrm{T}$ are identical. These tables are interesting and valuable, but for the particular purpose of finding the relation of $\theta$ and $\mathrm{T}$, we need similar but more detailed and exact values for such pressures as are used in gas-thermometers.

Equations (A), (B), and (C) show the relation of $\lambda$ to $\mu$ and $\rho$; i.e., how $\lambda$ might be computed from the results of either form of the plug experiment. I have not made any such computations, but it seems not beyond the bounds of possibility that with our present experimental refinements the quantity $\lambda$ might be measured directly.

$\S 7$. The two most interesting problems of thermodynamics at the present day are both concerned with the scale of temperature. The first is the performance of the plug experiment at very low temperatures. For it is a highly interesting question whether such temperatures as $1^{\circ .7}$ absolute, as given in a recent paper by Olszewski $\uparrow$, would really be anything like that if we could measure them on the thermodynamic scale. At present we cannot say whether they would or not.

The other problem is the purely theoretical one of inventing a radiation scale which shall be based on the two laws of thermodynamics in their accepted form, and on nothing else except experimental facts that we are sure of and familiar with. The Stefan-Boltzmann law comes nearest to this. Boltzmann's deduction depended ultimately on the assumption that Maxwell's distribution of stress in the electromagnetic field was the real one. As Maxwell's reasoning on this point does not pretend to be conclusive, Boltzmann's deduction of

* Jour, de Phys. (3) jii. p. 307 (1894).

$\dagger$ Annalen der Physik, 1905, no. 10. 
Stefan's law for the black body could also not be considered conclusive. This state of affairs has been changed since we know, from the brilliant experiments of Lebedew and of Nichols and Hull, that the light-pressure does exist and does have, very approximately at all events, the value deduced a priori from Maxwell's theory. It still remains, however, highly desirable that another absolute radiation scale should be devised, so that we may have another and independent check on our high temperature measurements. Planck's formula rests on too many assumptions to be satisfactory theoretically; and Wien's displacement law, while its theoretical foundation is nearly as good as that of the StefanBoltzmann law, does not, by itself, offer a good method from the point of view of experimental accuracy.

U.S. Department of Agriculture,

Washington, Jan. 17, 1906.

LXII. Some Measurements of Wave-Lengths with a Modified Apparatus. By Lord RAYleigh, O.M., Pres. R.S.*

A $S$ the result of discussions held during the last three or A four years, it seems to be pretty generally agreed that the use of the diffraction-grating in fundamental work must be limited to interpolation between standard wavelengths determined by other means. Even under the advantageous conditions rendered possible by Rowland's invention of the concave grating, allowing collimators and object-glasses to be dispensed with, the accuracy attained in comparisons of considerably differing wave-lengths is found to fall short of what had been hoped. I think that this disappointment is partly the result of exaggerated expectations, against which in $1888 \dagger$ I gave what was intended to be a warning. Quite recently, Michelson $\ddagger$ has shown in detail how particular errors of ruling may interfere with results obtained by the method of coincidences ; but we must admit that the discrepancies found by Kayser $\S$ in experiments specially designed to test this question, are greater than would have been anticipated.

Under these circumstances, attention has naturally been directed to interference methods, and especially to that so

* Communicated by the Author.

† Wave Theory, Enc. Brit.; Scientific Papers, iii. p. 111, footnote.

\$ Astro-physical Journal, xviii. p. 278 (1903).

\Zeitschrift fiir wiss. Photographie, Bd. ii. p. 49 (1904). 